\title{
Early secretory antigenic target 6 and culture filtrate protein 10 as diagnostic indicators in IgA nephropathy associated with renal tuberculosis
}

\section{Yong Fang}

Clinic and Research Center of Tuberculosis, Shanghai Key Lab of Tuberculosis, Shanghai Pulmona Hospital, Tongji University School of Medicine, Shanghai

Xiao-Fang You ( $\nabla$ youxiaofangfk@163.com )

Tongji University School of Medicine

\section{Qing-hui Wang}

Department of urology, changhai hospital naval medical university, Shanghai

Jun-hui Li

Department of Nephrology, The Sixth People's Hospital, Shanghai Jiao Tong University School of Medicine, Shanghai

\section{Research article}

Keywords: Renal tuberculosis, IgA nephropathy, ESAT-6, CFP-10, Diagnostic value

Posted Date: January 24th, 2020

DOI: https://doi.org/10.21203/rs.2.21801/v1

License: (1) This work is licensed under a Creative Commons Attribution 4.0 International License. Read Full License 


\section{Abstract}

Background: This study evaluated the diagnostic value of early secretory antigenic target 6 (ESAT-6) and culture filtrate protein 10 (CFP-10) in immunoglobulin A nephropathy (IgAN) associated with renal tuberculosis (RT).

Methods: Between January 2013 and January 2016, 40 patients with IgAN (IgAN group), 32 patients with RT (RT group), and 52 patients with IgAN associated with RT (IgAN + RT group) were selected for this study. A Tuberculin skin test (TST) was conducted, and serum Mycobacterium tuberculosis (MTB) antibody levels were measured. Urine samples were collected to culture MTB. Immunohistochemistry and western blotting were used to determine the expression of ESAT- 6 and CFP-10 in renal tissues. Receiver operating characteristic (ROC) analysis was used to evaluate the diagnostic values of ESAT- 6 and CFP-10 in IgAN associated with RT.

Results: TST, serum MTB antibody, and urine MTB assessments were negative in the IgAN group. The positive rates of the TST and serum MTB antibody and urine MTB testing were higher in the RT group than in the IgAN + RT group. Among the three groups, expression levels of ESAT- 6 and CFP-10 were found to be the highest in the IgAN + RT group and were found to be the lowest in the IgAN group. The ROC curves indicated that the area under curve (AUC) value of ESAT-6 protein for IgAN + RT diagnosis was 0.907 with a cut-off of 26.72 as the critical value. Detection by ESAT-6 protein levels achieved $75.0 \%$ sensitivity and $94.2 \%$ specificity. The AUC value of the CFP-10 protein for diagnosis of IgAN + RT was 0.800 , with a cut-off of 25.665 as the critical value. Detection by the protein levels of CFP-10 showed $63.9 \%$ sensitivity and $84.6 \%$ specificity.

Conclusions: Our study provides evidence for the potential of the proteins ESAT- 6 and CFP-10 as candidate markers for the diagnosis of IgAN associated with RT.

\section{Background}

Immunoglobulin A nephropathy (IgAN) is a type of glomerulonephritis that is common worldwide and usually affects people at a young age [1, 2]. IgAN is often associated with mucosal infections and is usually accompanied by recurrent episodes of gross haematuria, microscopic haematuria, or acute nephritic syndrome [3]. Current treatments for IgAN include antiplatelet medications, oral prednisolone, and tonsillectomy combined with steroid pulse therapy [4]. It is estimated that renal failure occurs in $40 \%$ of the IgAN patients within 20 years after diagnosis, and the long-term prognosis remains relatively poor [5]. Findings have also indicated that it is highly risky for patients to develop tuberculosis at an advanced stage of renal disease [6]. Renal tuberculosis (RT), caused by members of the Mycobacterium tuberculosis (MTB) complex, is a serious, localised genitourinary disease [7, 8]. Symptoms of RT include a high frequency of painless micturition and a series of pathological changes, from typical calcified lesions and papillary necrosis to autonephrectomy [9]. Other mild and non-specific symptoms include a small number of leukocytes in the urine and a low sedimentation rate [10]. Patients with RT do not 
usually experience the typical symptoms of tuberculosis [8]. Therefore, clinicians often find RT diagnosis quite challenging and find it difficult to prescribe the most suitable treatment for patients with these two diseases, which can lead to more severe renal failure [4].

Early secretory antigenic target 6 (ESAT-6) and culture filtrate protein 10 (CFP-10) are low-molecularweight proteins that are respectively encoded by the $R v 3874$ and $R v 3875$ genes located in the region of difference- 1 in the genome of virulent MTB strains $[11,12]$. The ESAT- 6 and the CFP-10 proteins can induce the malignant spread of MTB by participating in the cytolysis of alveolar epithelial cells and macrophages [13]. Recent evidence has also shown that when used together, the ESAT-6 and the CFP-10 antigens, two potential diagnostic markers, can also enhance the diagnostic specificity and sensitivity of tuberculosis testing [14]. Therefore, detecting the ESAT-6 and the CFP-10 proteins may allow early targeted diagnosis of RT and help to distinguish patients infected with MTB from individuals vaccinated with Bacillus Calmette Guerin [12]. Based on these findings, this study aimed to investigate the diagnostic value of the ESAT- 6 protein and the CFP-10 protein in IgAN associated with RT, which will be highly significant for diagnosing patients with these two diseases.

\section{Methods}

\section{Study subjects}

Between January 2013 and January 2016, 40 patients with IgAN (IgAN group), 32 patients with RT (RT group), and 52 patients with RT associated with IgAN (IgAN + RT group) diagnosed by renal biopsy were enrolled in this study. The age ranges of the patients in the three groups were 27 years- 54 years, 25 years -59 years, and 24 years -57 years old, respectively, and the male to female ratios were $21: 19,17: 15$, and 23:29, respectively. None of the patients had hepatitis, systemic lupus erythematosus, allergic purpura, or other secondary nephritis, and none had been administered any glucocorticoid and immunosuppressant before renal biopsy. Pure protein derivative of tuberculin (PPD) (the Shanghai Institute of Biological Products, Shanghai, China) was used for the tuberculin skin test (TST), which was conducted as follows: PPD was injected intracutaneously through the forearm-palmaris, and the injection site was observed for a reaction about $72 \mathrm{~h}$ after injection. Patients were considered to be PPD positive if the reaction was $>5 \mathrm{~mm}$ [15]. Venous blood $(3 \mathrm{~mL})$ was collected from all patients after fasting. Serum was isolated from the blood samples and used for the detection of anti-tuberculosis antibody.

\section{Urine culture for MTB}

Urinary sediment $(20 \mathrm{~mL})$ was obtained from 24 -h urine specimens and then centrifuged to obtain the precipitate. The sediment was mixed with $4 \%$ sodium hydroxide $(\mathrm{NaOH})$ to facilitate the precipitation. The supernatant was removed and centrifuged under sterile conditions, and the precipitate was collected and mixed with $6 \%$ sulphuric acid. The specimens were then inoculated on a Roche slanted solid culture medium and incubated in a constant temperature incubator at $37^{\circ} \mathrm{C}$ and observed at regular time intervals. After 2 months of observation, bacterial growth on the culture medium indicated the presence of MTB. 


\section{Haematoxylin and eosin (HE) staining}

Renal tissue specimens were fixed with $4 \%$ paraformaldehyde, washed with phosphate buffered saline (PBS), dehydrated with graded ethanol, and cleared with xylene. After the remaining xylene was washed away with distilled water, the tissue specimens were embedded in paraffin, sliced into 4- $\mu \mathrm{m}$ sections, and deparaffinised with ethanol. Some sections were stained with HE. Briefly, prepared renal tissue sections were stained with haematoxylin for 5 min, washed with distilled water, stained with $0.5 \%$ eosin for 2 min, dehydrated with ethanol, cleared in xylene, sealed with neutral gum, and then observed with an optical microscope. The remaining sections were stored at $-20^{\circ} \mathrm{C}$ until use.

\section{Immunohistochemistry}

After adding $30 \% \mathrm{H}_{2} \mathrm{O}_{2}$ to block endogenous enzyme activity, renal tissue sections from the patients were heated in antigen retrieval buffer. After cooling for $5 \mathrm{~min}$, the sections were heated and cooled twice more. After cooling to a temperature that coincided with the room temperature (about $25^{\circ} 5$ ), the sections were blocked by adding $5 \%$ bovine serum albumin (BSA) blocking buffer and incubating at room temperature for $20 \mathrm{~min}$. The excess blocking buffer was removed, and then mouse anti-human ESAT-6 primary antibody (ab26246, 1:3000; Abcam, Cambridge, MA, USA) and mouse anti-human CFP-10 primary antibody (ab64754, 1:3000; Abcam) were added to the sections and incubated at $4^{\circ} \mathrm{C}$ overnight. Then, the sections were incubated with biotinylated goat-anti mouse lgG (ab6789; Abcam) at $37^{\circ} \mathrm{C}$ for $40 \mathrm{~min}$. The sections were washed with PBS, and the antibody-antigen reactions were visualised by incubation with 3,3'-diaminobenzidine; Beijing Zhongshan Jinqiao Biotechnology Co., Ltd., Beijing, China). Images of the stained sections (three randomly selected fields from three sections of each specimen) were quantitatively analysed with the Image-ProPlus (Media Cybernetics, Rockville, MD, USA) to determine the integral optical density (IOD) values of the ESAT-6 protein and the CFP-10 protein.

\section{Western blotting}

The renal tissue sections were incubated with $1 \mathrm{X}$ sodium dodecyl sulphate buffer with mixing at $300 \times \mathrm{g}$ until the sections were fully lysed. The lysed sections were then incubated on ice for $30 \mathrm{~min}$ and centrifuged for $4 \mathrm{~min}$ at $1200 \times \mathrm{g}$. The supernatant was removed and stored at $-80^{\circ} \mathrm{C}$. The concentration of the extracted proteins in the supernatant was determined using the BCA protein determination kit (AR0146; Boster Biological Technology Co., Ltd., Wuhan, China), which was then adjusted to $3 \mu \mathrm{g} / \mu \mathrm{L}$. The protein samples were mixed with loading buffer and incubated for $10 \mathrm{~min}$ at $95^{\circ} \mathrm{C}$, and then equal protein $(30 \mu \mathrm{g})$ from each sample was separated by $10 \%$ polyacrylamide gel electrophoresis. The separated proteins were then transferred to a PVDF membrane (P2438; Sigma, St. Louis, MO, USA) by semi-dry electrotransfer and then blocked with $5 \% \mathrm{BSA}$ for $1 \mathrm{~h}$ at room temperature. Then, the membrane was incubated with anti-ESAT- 6 antibody (ab45073, 1:1000 in 5\% BSA; Abcam) or rabbit anti-CFP-10 (ab45074, Abcam) and rabbit anti-a-smooth muscle actin (ab5694; Abcam), which was diluted to 0.2

$\mu \mathrm{g} / \mathrm{mL}-1 \mu \mathrm{g} / \mathrm{mL}$, in a refrigerator with shaking overnight. The membranes were washed three times with TBST for 10 min each and then incubated with the secondary antibody (ab6720, 1:10000; Abcam) in 
TBST for $4 \mathrm{~h}-6 \mathrm{~h}$ at $4^{\circ} \mathrm{C}$. After incubation, the membrane was washed with TBST three times for 15 min each. The membrane was then incubated with chemiluminescence reagents $A$ and $B$, mixed at 1:1, followed by the addition of photographic developer solutions. GAPDH was used as an internal reference. All immunoblotting bands were subjected to densitometric analysis.

\section{Immunofluorescence}

Renal tissue sections from patients were heated to a boil in antigen retrieval buffer. After cooling for 5 min, the sections were heated and cooled two more times, and then cooled to room temperature. Then, the sections were incubated with mouse anti-human IgA1-FITC (ab99793, 1:50; Abcam) in a humidified chamber at $37^{\circ} \mathrm{C}$ for $30 \mathrm{~min}$. The sections were washed with PBS (pH 7.2- $\left.\mathrm{pH} 7.6\right)$ three times for $5 \mathrm{~min}$ each. After air drying, the sections were sealed with buffered glycerol and observed with a fluorescence microscope under a glass coverslip. Five high-power fields (400X) of each section were randomly selected, and the corresponding mean gray value (MGV) of the positively-stained areas was measured.

\section{Statistical analysis}

All data were analysed with SPSS 21.0 (SPSS, Inc., Chicago, IL, USA). Data have been presented as the mean \pm standard deviation. The $t$-test was used for comparisons between two groups, and one-way analysis of variance (ANOVA) was used for comparisons of multiple groups. Counted data have been expressed as a rate or percentage, and two groups were compared using the chi-square test. For multiple groups with equal variance, the $q$ test was performed for pairwise comparisons, and the non-parametric rank test was performed for group comparisons. A receiver operating characteristic (ROC) curve $(a=0.05)$ was plotted to evaluate the diagnostic value of the ESAT- 6 and the CFP-10 proteins. $P$ values less than 0.05 were regarded as statistically significant.

\section{Results}

\section{More IgAN + RT patients had a positive TST and were positive for serum MTB antibody}

There was no significant difference in the gender or age of the patients in the IgAN, RT, and IgAN + RT groups ( $P>0.05$ for both). Patients in the IgAN group had no history of tuberculosis, and patients in the RT group had no history of renal disease. No significant difference was noted in the history of tuberculosis between the RT and IgAN + RT groups $(P=0.390)$ or the history of renal disease between the IgAN and IgAN + RT groups $(P=0.124)$. The TSTs and serum tuberculosis antibody tests of patients in the IgAN group were negative. There were $22(68.75 \%)$ patients and $38(73.08 \%)$ patients in the RT and IgAN + RT groups, respectively, who had a positive TST; and $23(31.94 \%)$ patients and 41 (78.85\%) patients in the RT and IgAN + RT groups, respectively, with positive serum tuberculosis antibody tests. The positive rates for the TST and serum tuberculosis antibody test in the RT and IgAN + RT groups were both higher than those in the IgAN group $(P<0.05$ for both; Table 1$)$.

\section{More IgAN + RT patients were positive for MTB in urine culture}


There were $10(31.2 \%)$ patients and $30(57.7 \%)$ patients who showed positive results for MTB in the urine culture test in the RT and IgAN + RT groups, respectively (Table 2). Compared with the IgAN group, the positive rates in both the RT and IgAN + RT groups were significantly higher. However, the rate in the IgAN $+\mathrm{RT}$ group was found to be significantly higher than that in the RT group $(P<0.05)$.

\section{Pathological changes in the renal tissues of patients in the IgAN, RT, and IgAN + RT groups}

Figure 1 shows the following pathological changes in the patient groups. In the IgAN group, cellular crescents had formed in the renal tissues, the glomerular mesangium was found to be thickened, and the amount of mesangial matrix and glomerular mesangial cells were found to be increased. In the RT group, tubercles were observed with caseous necrosis at the centre, along with Langerhans cells, epithelioid cells, and a large number of lymphocytes. In the IgAN + RT group, segments, moderate mesangial cell proliferation, and increased mesangial matrix were observed.

\section{IgAN + RT patients showed high IOD values for the ESAT-6 and the CFP-10 proteins}

In the RT and IgAN + RT groups, yellowish-brown and brown positive deposits were observed in the renalinterstitium, glomerular mesangial region, and cytoplasm of the kidney tubule cells. In the IgAN group, only two sections had light-brown deposits in the renal-interstitium. Pairwise comparisons were performed of the IgAN, RT, and IgAN + RT groups, which showed that the IOD values of the ESAT- 6 protein and the CFP-10 protein in the renal tissues of patients were found to be the highest in the $\operatorname{lgAN}+\mathrm{RT}$ group and the lowest in the IgAN group $(P<0.05$ for both; Figure 2$)$.

\section{IgAN + RT patients showed high levels of the ESAT-6 and the CFP-10 proteins}

Pairwise comparisons of the IgAN, RT, and IgAN + RT groups showed that the EAST- 6 and the CFP-10 protein levels in the renal tissues were found to be the highest in the $\lg A N+R T$ group and the lowest in the IgAN group $(P<0.05$ for both; Figure 3$)$.

\section{Enhanced IgA1 expression (visible as fluorescence) was detected in the renal tissues of patients in the $\operatorname{IgAN}$ and IgAN + RT groups}

Immunofluorescence detection showed that there were yellow-green deposits of IgA1 in the glomerular mesangium, capillary loop, and a portion of the renal tubular epithelial cells in the IgAN and IgAN + RT groups. In contrast, the renal tissues of patients in the RT group were found to be negative for IgA1 expression. The MGVs in the IgAN, IgAN + RT, and RT groups were $57.83 \pm 7.16,55.74 \pm 6.08$, and $10.02 \pm$ 1.38 , respectively. Compared with the RT group, both the IgAN and IgAN + RT groups showed significantly higher IgA1 expression ( $P<0.05$ for both). However, IgA1 expression levels in the renal tissues of patients did not differ significantly between the $\lg \mathrm{AN}$ and $\lg \mathrm{AN}+\mathrm{RT}$ groups $(P>0.05$; Figure 4$)$.

The ESAT-6 and the CFP-10 proteins showed good diagnostic performance for IgAN associated with RT 
Based on the ROC curves, the area under curve (AUC) of the ESAT-6 protein for IgAN + RT diagnosis was $0.907(95 \% \mathrm{Cl}=0.852-0.963, P<0.001)$, the cut-off value was 26.72 , and the sensitivity and specificity were $75.0 \%$ and $94.2 \%$, respectively; the AUC of the CFP-10 protein for IgAN + RT diagnosis was 0.800 $(95 \% \mathrm{Cl}=0.721-0.880, P<0.001)$, the cut-off value was 25.665 , and the sensitivity and specificity were found to be $63.9 \%$ and $84.6 \%$, respectively (Figure 5 ).

\section{Discussion}

RT is an infectious disease caused by MTB strains [7]. IgAN, which is one of the most common primary glomerular diseases in young males worldwide, is particularly prevalent in Asia [16]. Although many proteins have been used to improve the diagnosis and prognosis of RT over the past few years, and a renal biopsy is necessary to diagnose $\lg A \mathrm{~N}$, numerous clinical studies have suggested that new tools are still urgently needed to distinguish IgAN and RT [14, 17-19]. In this study, we investigated whether the expression of the ESAT- 6 and CFP-10 proteins could serve as diagnostic indicators of IgAN associated with RT. The results showed that ESAT- 6 and CFP-10 have high value for the diagnosis of IgAN associated with RT.

Initially, our study showed that, compared with the IgAN group, the RT and IgAN + RT groups had higher MTB urine culture positive rates, and the positive rate in the IgAN + RT group was higher than that in the RT group. This result indicated that the rates of MTB detection were much higher in patients with RT than in patients without RT. This corresponded to a study showing that, under most circumstances, RT was caused by MTB [8]. However, the IgAN + RT group showed a higher MTB positive rate than the RT group. Previous studies have suggested that the immune complexes, such as IgA antibodies, in IgAN patients might contribute to the response to MTB, which was further confirmed by our results [20, 21].

Our immunohistochemistry analysis showed that the IOD values of The ESAT- 6 and the CFP-10 proteins were highest in the IgAN + RT group, followed by the RT and IgAN groups, respectively. T cell-mediated immunity is critical to control MTB infections. The ESAT- 6 and the CFP-10 proteins are good candidates for stimulating T cells in MTB infected tissues to stimulate the production of interferon- $\gamma$ and other cytokines, such as IL-2 $[22,23]$. It has been reported that cells harvested from the lungs and Peyer's patches of immunised mice stimulated with the CFP-10 protein exhibit significant IFN- $y$ levels and these mice produce increased levels of serum IgG and lung IgA anti-CFP-10 responses [24]. In addition, and this research supported the potential of an IgA response against the ESAT-6/CFP-10 antigens for discriminating clinical TB from healthy MTB-infected and uninfected cases. The kidney is one of the main sites of tuberculosis infection, as it precisely meets the stringent growth requirements of $M$. tuberculosis due to its high oxygen tension. Genitourinary tuberculosis, including RT, is the second most common extrapulmonary tuberculosis infection after lymphatic tuberculosis $[25, \underline{26}]$. Therefore, the two proteins showed stronger reactions in the IgAN + RT and IgAN groups. In light of the fact that renal insufficiency is a risk factor for MTB infection [27], it is not surprising that patients in the IgAN + RT group with more severe renal damage had a higher level of MTB. Furthermore, a previous study demonstrated that both ESAT-6 and CFP-10, which are produced by the ESX-1 system in MTB, play a significant role in 
the development and spread of MTB infection [13]. Thus, the presence of MTB in the RT and IgAN + RT groups facilitated the response to the ESAT- 6 and the CFP-10 antibodies, thus leading to higher IOD values.

Furthermore, our results showed that IgA1 expression levels were much higher in the $\lg A \mathrm{~N}$ and $\lg \mathrm{AN}+\mathrm{RT}$ groups than in the RT group. IgA1 is a unique serum glycoprotein, which is a subclass of IgA that exclusively exists in the mesangial deposits in patients with $\operatorname{IgAN}[28,29]$. Another study mentioned that galactose-deficient IgA1 is one of the most accurate indicators for the pathogenesis of IgAN [30]. In terms of the relevant mechanism, deposits of IgA1 molecules likely play an active role in causing renal damage by interacting directly with resident glomerular cells and regulating complement activation [31]. Hence, IgA1 expression was weaker in the RT group than in the IgAN and IgAN + RT groups.

\section{Conclusions}

In summary, the present study provides evidence that both the ESAT- 6 protein and the CFP-10 protein are of high value for diagnosing IgAN accompanied by RT. The expression levels of these two proteins, as demonstrated by our results, were found to be the highest in IgAN + RT patients, indicating their great potential for the diagnosis of renal diseases and exploring the pathogenesis of these diseases. Since this study involved a relatively small sample, further large-scale trials will be required to confirm this conclusion.

\section{List Of Abbreviations}

CFP-10, culture filtrate protein 10; ESAT-6, early secretory antigenic target 6; IgAN, immunoglobulin A nephropathy; MTB, Mycobacterium tuberculosis; TST, Tuberculin skin test; RT, renal tuberculosis; ROC, Receiver operating characteristic; AUC, area under curve; HE, Haematoxylin and eosin; PBS, phosphate buffered saline; BSA, bovine serum albumin; IOD, integral optical density; ANOVA, analysis of variance; $M G V$, mean gray value.

\section{Declarations}

\section{Ethics approval and consent to participate}

This study was approved by the Ethics Committee of the Shanghai Pulmonary Hospital, and all patients provided informed consent.

\section{Consent for publication}

Not applicable.

\section{Availability of data and materials}


The datasets analysed during the current study are available from the corresponding author on reasonable request.

\section{Competing interests}

The authors declare that they have no competing interests.

\section{Funding}

None.

\section{Authors' contributions}

Xiao-Fang You designed the experiments. Yong Fang carried out the majority of experiments and wrote the article. Qing-hui Wang and Jun-hui Li revised the article. All authors read and approved the final manuscript.

\section{Acknowledgements}

Not applicable.

\section{References}

1. Kawamura T, Yoshimura M, Miyazaki Y, Okamoto H, Kimura K, Hirano K, Matsushima M, Utsunomiya $\mathrm{Y}$, Ogura M, Yokoo T et al: A multicenter randomized controlled trial of tonsillectomy combined with steroid pulse therapy in patients with immunoglobulin A nephropathy. Nephrology, dialysis, transplantation : official publication of the European Dialysis and Transplant Association - European Renal Association 2014, 29(8):1546-1553.

2. Appel GB, Waldman M: The IgA nephropathy treatment dilemma. Kidney internationa/2006, 69(11):1939-1944.

3. Kiryluk K, Novak J, Gharavi AG: Pathogenesis of immunoglobulin A nephropathy: recent insight from genetic studies. Annual review of medicine 2013, 64:339-356.

4. Imai $\mathrm{H}$, Miura $\mathrm{N}$ : A treatment dilemma in adult immunoglobulin A nephropathy: what is the appropriate target, preservation of kidney function or induction of clinical remission? Clinical and experimental nephrology 2012, 16(2):195-201.

5. Kaneko T, Hirama A, Ueda K, Fujino T, Utsumi K, lino Y, Katayama Y: Methylprednisolone pulse therapy combined with mizoribine following tonsillectomy for immunoglobulin A nephropathy: clinical remission rate, steroid sparing effect, and maintenance of renal function. Clinical and experimental nephrology 2011, 15(1):73-78.

6. Winthrop KL, Nyendak M, Calvet H, Oh P, Lo M, Swarbrick G, Johnson C, Lewinsohn DA, Lewinsohn $\mathrm{DM}$, Mazurek GH: Interferon-gamma release assays for diagnosing mycobacterium tuberculosis 
infection in renal dialysis patients. Clinical journal of the American Society of Nephrology : CJASN 2008, 3(5):1357-1363.

7. Chaudhari AP, Ranganath R, Pavan M: Unusual presentation of renal tuberculosis. Iranian journal of kidney diseases 2011, 5(3):207-209.

8. Daher Ede F, da Silva GB, Jr., Barros EJ: Renal tuberculosis in the modern era. The American journal of tropical medicine and hygiene 2013, 88(1):54-64.

9. Dharmapalan A, Vijaykumar R, Bhoopal S: Renal tuberculosis presenting as thigh abscess. The Indian journal of surgery 2013, 75(Suppl 1):446-448.

10. Lattimer JK, Vasquez G: Renal tuberculosis in children. Postgraduate medicine 1960, 28:336-342.

11. Jackson-Sillah D, Cliff JM, Mensah GI, Dickson E, Sowah S, Tetteh JK, Addo KK, Ottenhoff TH, Bothamley G, Dockrell HM: Recombinant ESAT-6-CFP10 Fusion Protein Induction of Th1/Th2 Cytokines and FoxP3 Expressing Treg Cells in Pulmonary TB. PloS one 2013, 8(6):e68121.

12. Tang XL, Zhou YX, Wu SM, Pan Q, Xia B, Zhang XL: CFP10 and ESAT6 aptamers as effective Mycobacterial antigen diagnostic reagents. The Journal of infection 2014, 69(6):569-580.

13. Xie X, Han M, Zhang L, Liu L, Gu Z, Yang M, Yang H: Effects of Mycobacterium tuberculosis ESAT6CFP10 Protein on Cell Viability and Production of Nitric Oxide in Alveolar Macrophages. Jundishapur journal of microbiology 2016, 9(6):e33264.

14. van Pinxteren LA, Ravn P, Agger EM, Pollock J, Andersen P: Diagnosis of tuberculosis based on the two specific antigens ESAT-6 and CFP10. Clinical and diagnostic laboratory immunology 2000, 7(2):155-160.

15. Brabin L, Brabin BJ, Crane G: Difficulties of interpreting PPD reactions of women living in Madang, Papua New Guinea. Transactions of the Royal Society of Tropical Medicine and Hygiene 1994, 88(3):349-353.

16. Tipu HN, Ahmed TA, Bashir MM: Clinical, histopathological and immunofluorescent findings of IgA nephropathy. Iranian journal of immunology : IJI2011, 8(2):104-110.

17. Kiryluk K, Julian BA, Wyatt RJ, Scolari F, Zhang H, Novak J, Gharavi AG: Genetic studies of IgA nephropathy: past, present, and future. Pediatr Nephrol 2010, 25(11):2257-2268.

18. Berthoux FC, Mohey H, Afiani A: Natural history of primary IgA nephropathy. Seminars in nephrology 2008, 28(1):4-9.

19. Wang J, Fan S, Xiao J, Liang C: Renal tuberculosis tends to be low symptoms: how to improve the diagnosis and treatment of renal tuberculosis. Asian Journal of Andrology 2016, 18(1):145-146.

20. Chan JC, Trachtman H: Modulating the progression in IgA nephropathy. Nephron Clinical practice 2006, 104(1):c61-68.

21. Bezerra JM, Beck ST, Kanunfre KA, Leite OM, Ferreira AW: A study of IgA antibody response to different mycobacterium tuberculosis antigens in the diagnosis and monitoring of pulmonary tuberculosis. The Brazilian journal of infectious diseases : an official publication of the Brazilian Society of Infectious Diseases 2009, 13(1):53-58. 
22. Arend SM, de Haas P, Leyten E, Rosenkrands I, Rigouts L, Andersen P, Mijs W, van Dissel JT, van Soolingen D: ESAT-6 and CFP-10 in clinical versus environmental isolates of Mycobacterium kansasii. The Journal of infectious diseases 2005, 191(8):1301-1310.

23. Arlehamn CSL, Sidney J, Henderson R, Greenbaum JA, James EA, Moutaftsi M, Coler RN, Mckinney DM, Park D, Taplitz R: Dissecting Mechanisms of Immunodominance to the Common Tuberculosis Antigens ESAT-6, CFP10, Rv2031c (hspX), Rv2654c (TB7.7), and Rv1038c (EsxJ). Journal of Immunology 2012, 188(10):5020-5031.

24. Zhang H, Wen K, Shen J, Geng S, Huang J, Pan Z, Jiao X: Characterization of immune responses following intranasal immunization with the Mycobacterium bovis CFP-10 protein expressed by attenuated Salmonella typhimurium. Scandinavian journal of immunology 2010, 72(4):277-283.

25. Colbert G, Richey D, Schwartz JC: Widespread tuberculosis including renal involvement. Proc (Bay/ Univ Med Cent) 2012, 25(3):236-239.

26. Bhatt C, Lodha S: Paraspinal sinuses? Do remember renal tuberculosis. Case Reports 2012, 2012.

27. Li SY, Chen TJ, Chung KW, Tsai LW, Yang WC, Chen JY, Chen TW: Mycobacterium tuberculosis infection of end-stage renal disease patients in Taiwan: a nationwide longitudinal study. Clinical microbiology and infection : the official publication of the European Society of Clinical Microbiology and Infectious Diseases 2011, 17(11):1646-1652.

28. Hiki Y, Odani H, Takahashi M, Yasuda Y, Nishimoto A, Iwase H, Shinzato T, Kobayashi Y, Maeda K: Mass spectrometry proves under-0-glycosylation of glomerular IgA1 in IgA nephropathy. Kidney international 2001, 59(3):1077-1085.

29. Hiki Y, Kokubo T, Iwase H, Masaki Y, Sano T, Tanaka A, Toma K, Hotta K, Kobayashi Y:

Underglycosylation of IgA1 hinge plays a certain role for its glomerular deposition in IgA nephropathy. Journal of the American Society of Nephrology : JASN 1999, 10(4):760-769.

30. Yasutake J, Suzuki Y, Suzuki H, Hiura N, Yanagawa H, Makita Y, Kaneko E, Tomino Y: Novel lectinindependent approach to detect galactose-deficient IgA1 in IgA nephropathy. Nephrology, dialysis, transplantation : official publication of the European Dialysis and Transplant Association - European Renal Association 2015, 30(8):1315-1321.

31. Giannakakis K, Feriozzi S, Perez M, Faraggiana T, Muda AO: Aberrantly glycosylated IgA1 in glomerular immune deposits of IgA nephropathy. Journal of the American Society of Nephrology : JASN 2007, 18(12):3139-3146.

\section{Tables}

Table 1 Comparisons of baseline characteristics of patients in IgAN, RT and IgAN + RT groups 


\begin{tabular}{llll}
\hline Characteristic & IgAN group & RT group & IgAN + RT group \\
& $(\mathrm{n}=40)$ & $(\mathrm{n}=32)$ & $(\mathrm{n}=52)$ \\
\hline
\end{tabular}

Gender

$\begin{array}{rrrr}\text { Male } & 21 & 17 & 23 \\ \text { Female } & 19 & 15 & 29\end{array}$
Age (year)
$40.70 \pm 9.23 \quad 40.66 \pm 10.70$
$42.27 \pm 8.51$

History of tuberculosis (month)

$0^{\mathrm{a}} \quad 4.17 \pm 1.93^{\mathrm{b}} \quad 4.60 \pm 1.85^{\mathrm{b}}$

History of renal disease (month)

$2.10 \pm 0.99^{a}$

$0^{\mathrm{b}}$

$1.73 \pm 0.82^{b}$

Tuberculin skin test

$\begin{array}{rrrr}\text { Positive } & 0 & 22 & 38 \\ \text { Negative } & 40^{\mathrm{a}} & 10^{\mathrm{b}} & 14^{\mathrm{c}}\end{array}$

Serum tuberculosis antibody

$\begin{array}{cccc}\text { Positive } & 0 & 23 & 41 \\ \text { Negative } & 40^{\mathrm{a}} & 9^{\mathrm{b}} & 11^{\mathrm{c}}\end{array}$

Notes: IgAN, immunoglobulin A nephropathy; RT, renal tuberculosis; a, b, and c letters means $P<0.05$ when pair comparisons were performed among the IgAN, RT, and IgAN + RT groups.

Table 2 Positive rates of urine $M T B$ in IgAN, RT and IgAN + RT groups 


\begin{tabular}{lllll}
\hline Group & \multicolumn{2}{l}{$M T B$ in urine } & \multicolumn{2}{l}{ Total Positive rate } \\
\cline { 2 - 3 } & \multicolumn{2}{l}{ Negative } & Positive & \\
\hline IgAN group & 40 & 0 & 40 & $0^{\mathrm{a}}$ \\
RT group & 22 & 10 & 32 & $31.2 \%^{\mathrm{b}}$ \\
IgAN + RT group & 22 & 30 & 52 & $57.7 \%^{\mathrm{c}}$ \\
\hline
\end{tabular}

Notes: MTB, mycobacterium tuberculosis; IgAN, immunoglobulin A nephropathy; RT, renal tuberculosis; a, b, and c letters means $P<0.05$ when pair comparisons were performed among the IgAN, RT, and IgAN + RT groups.

\section{Figures}
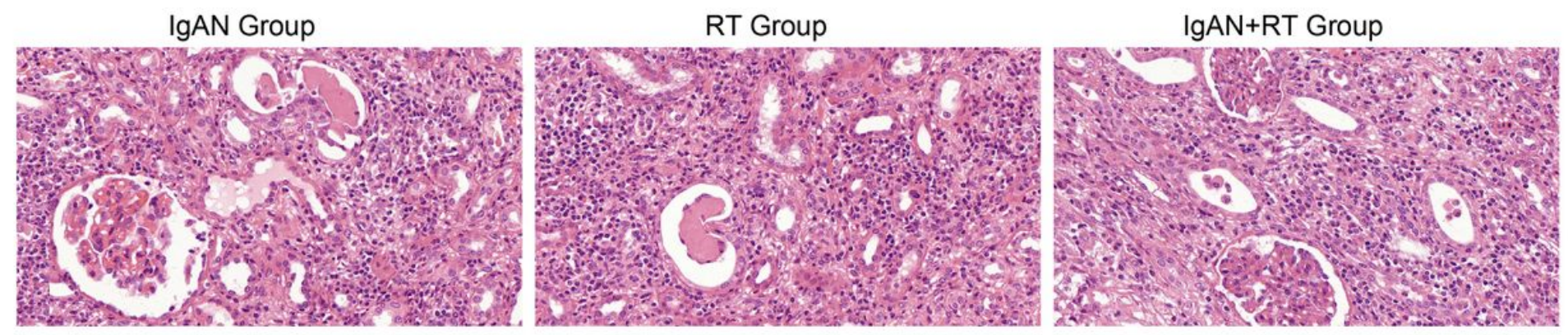

Figure 1

Pathological changes in the renal tissues of patients in the IgAN, RT, and IgAN + RT groups by HE staining (400X). Abbreviations: IgAN, immunoglobulin A nephropathy; RT, renal tuberculosis; $\mathrm{HE}$, haematoxylin and eosin. 

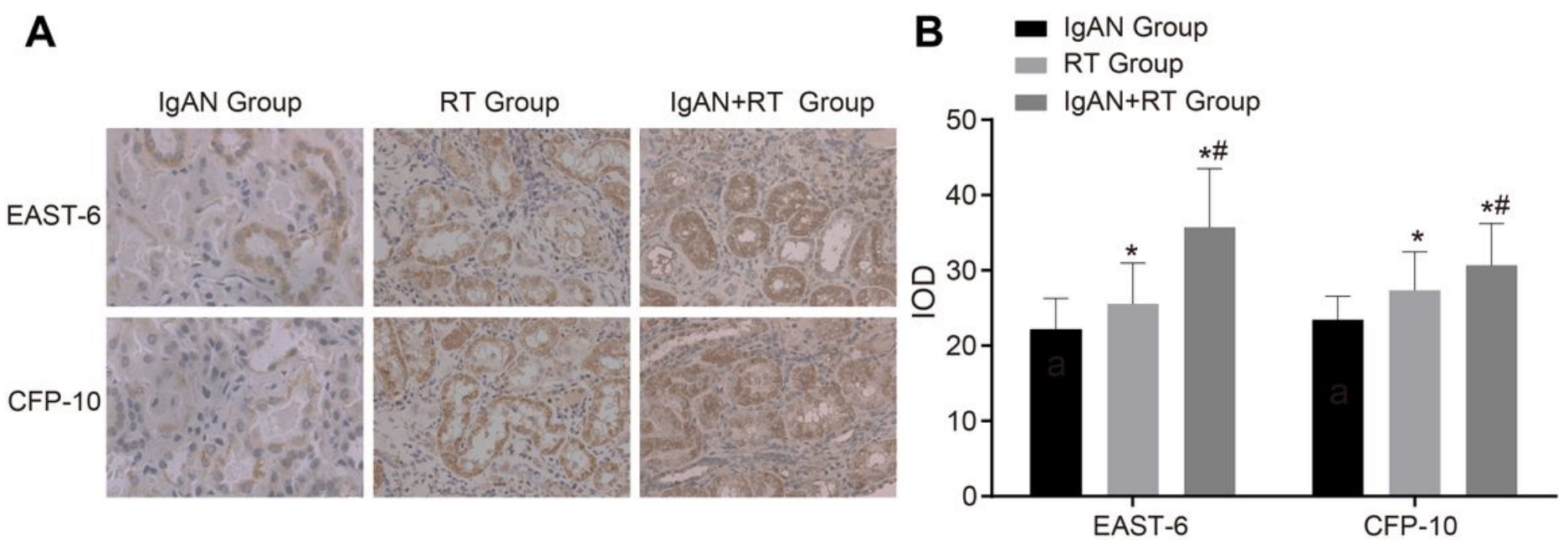

\section{Figure 2}

2 Immunohistochemical detection of the ESAT- 6 protein and the CFP-10 protein in the renal tissues of patients in the IgAN, RT, and IgAN + RT groups. A, Immunohistochemistry map of expressions of the ESAT-6 protein and the CFP-10 protein in each group; B, histogram of the IOD of the ESAT- 6 protein and the CFP-10 protein in each group. Abbreviations: ESAT-6, early secretory antigenic target 6; CFP-10, culture filtrate protein 10; IgAN, immunoglobulin A nephropathy; RT, renal tuberculosis; IOD, integral optical density; $a, b$, and $c$ indicate significant differences $(P<0.05)$ when pairs of groups (IgAN, RT, and IgAN + RT) were compared.

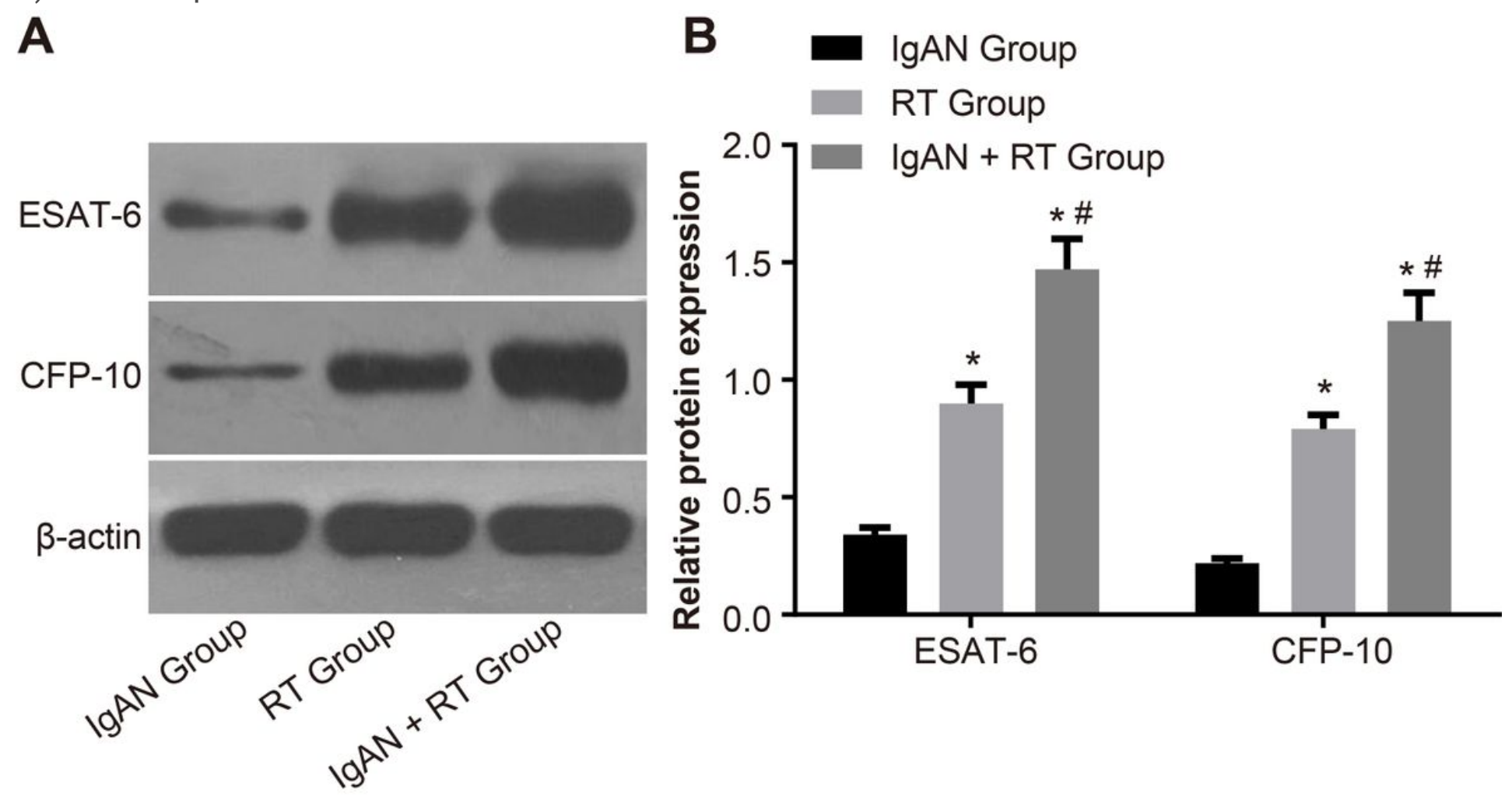

Figure 3 
Expressions of the ESAT- 6 protein and the CFP-10 protein in the renal tissues of patients in the IgAN, RT, and IgAN + RT groups as detected by western blotting. A, grey value of the protein bands of the ESAT- 6 protein and the CFP-10 protein; B, protein levels of ESAT- 6 and CFP-10. $a$, b, and c indicate significant differences $(P<0.05)$ when pairs of groups (IgAN, RT, and IgAN + RT) were compared.

\section{A}
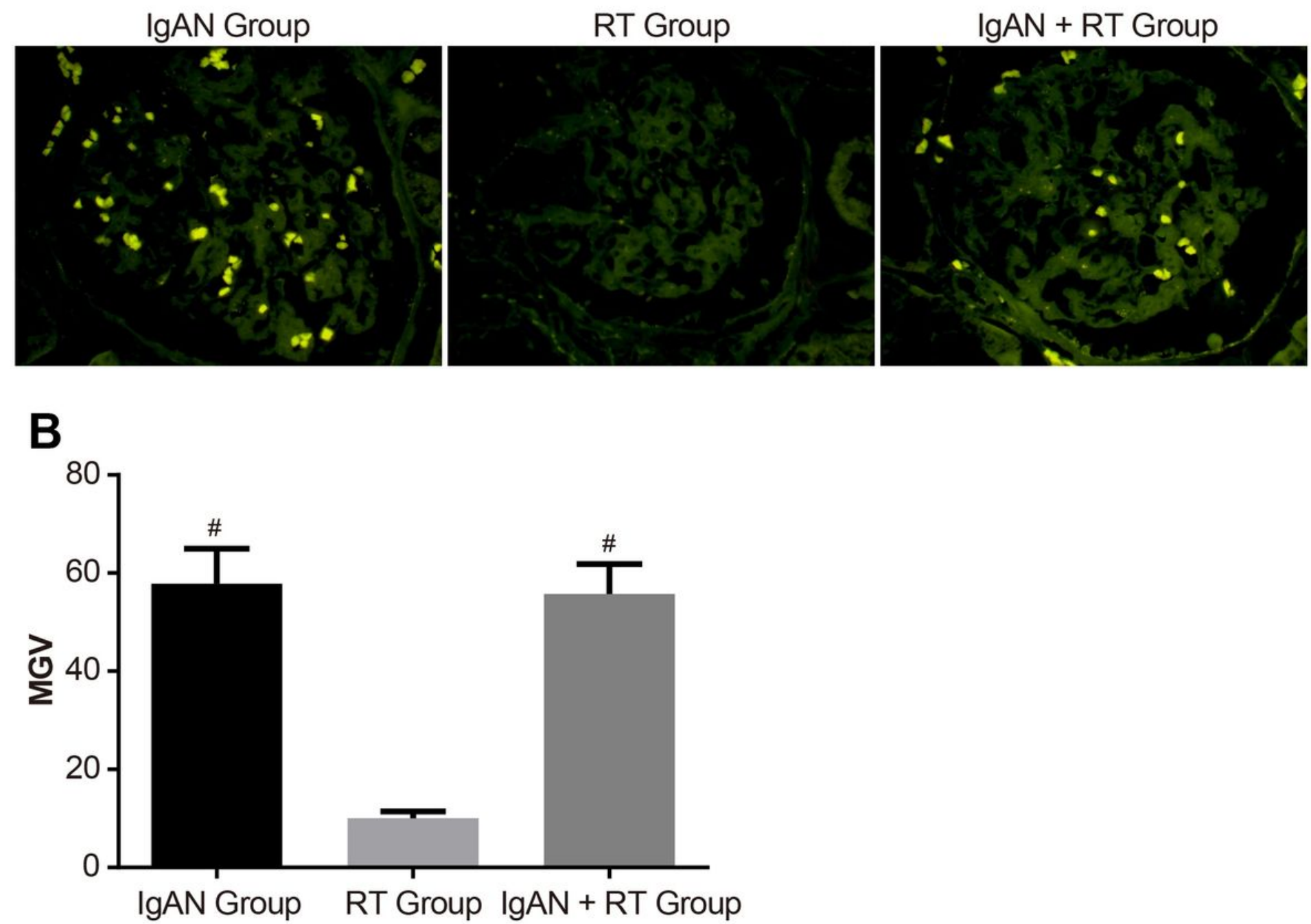

\section{Figure 4}

IgA1 expression in the renal tissues and the MGV in the IgAN, RT, and IgAN + RT groups. A, immunofluorescence images of IgA1 expression in the renal tissues of each group; $B$, histogram of the MGV in each group. Abbreviations: IgAN, immunoglobulin A nephropathy; RT, renal tuberculosis; MGV, mean gray value; IgA 1 , immunoglobulin $A 1$. a, b, and c indicate significant differences $(P<0.05)$ when pairs of groups (IgAN, RT, and IgAN + RT) were compared. 


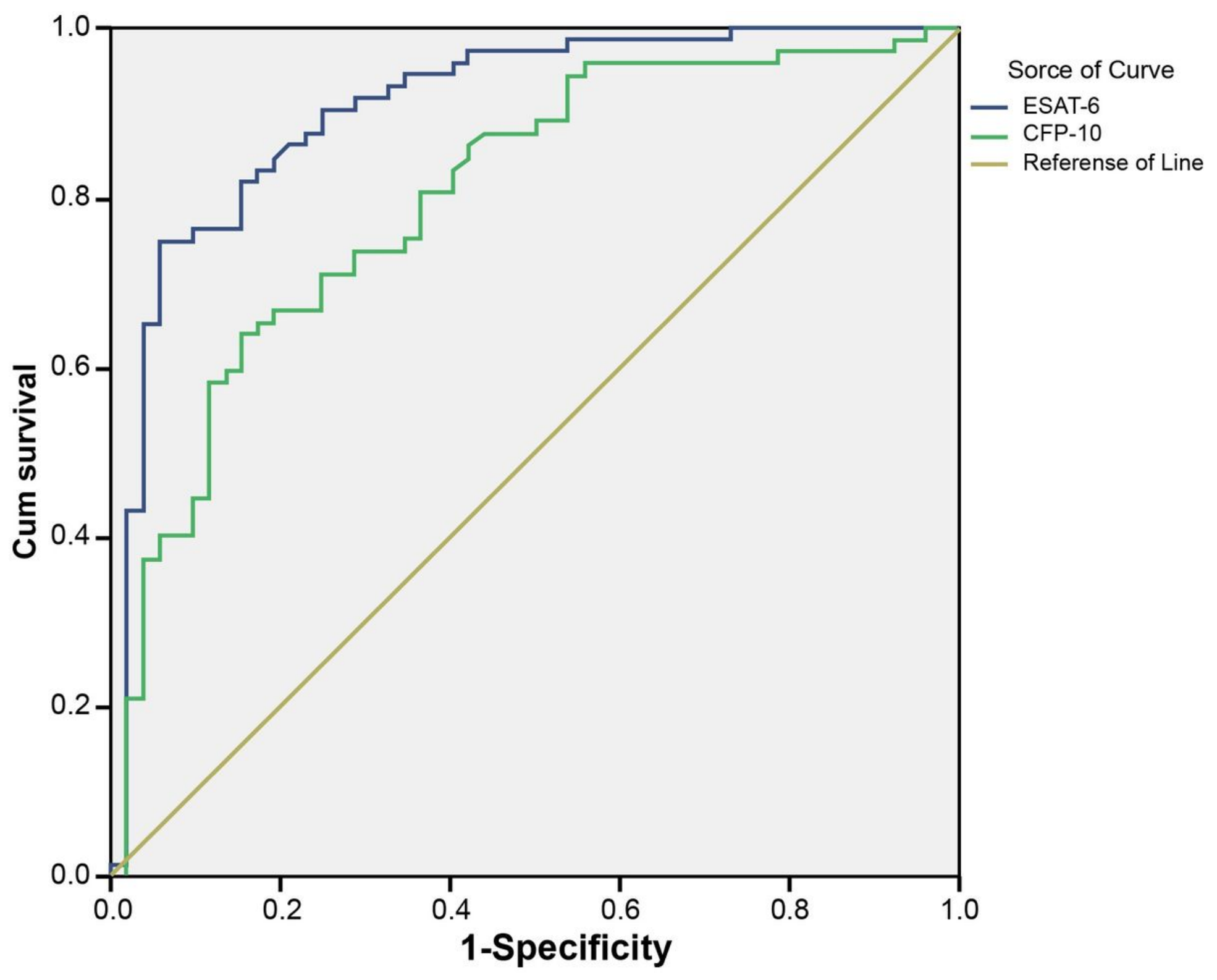

Figure 5

Diagnostic values of the ESAT-6 protein and the CFP-10 protein, as suggested by ROC curves, for the diagnosis of RT with IgAN. Abbreviations: ROC, receiver operating characteristic; ESAT-6, early secretory antigenic target 6; CFP-10, culture filtrate protein 10; IgAN, immunoglobulin A nephropathy; RT, renal tuberculosis. 